\title{
Integrated X-Ray-Scattering Intensity Measurement of the Order Parameter at the Nematic-to-Smectic-A Phase Transition
}

\section{Citation}

Chan, Kelby K., Moshe Deutsch, B. M. Ocko, Peter S. Pershan, and L. B. Sorensen. 1985. Integrated $\mathrm{x}$-ray-scattering intensity measurement of the order parameter at the nematic-tosmectic-A phase transition. Physical Review Letters 54(9): 920-923.

\section{Published Version}

doi:10.1103/PhysRevLett.54.920

\section{Permanent link}

http://nrs.harvard.edu/urn-3:HUL.InstRepos:10357694

\section{Terms of Use}

This article was downloaded from Harvard University's DASH repository, and is made available under the terms and conditions applicable to Other Posted Material, as set forth at http:// nrs.harvard.edu/urn-3:HUL.InstRepos:dash.current.terms-of-use\#LAA

\section{Share Your Story}

The Harvard community has made this article openly available.

Please share how this access benefits you. Submit a story.

\section{Accessibility}




\title{
Integrated X-Ray-Scattering Intensity Measurement of the Order Parameter at the Nematic-to-Smectic- $A$ Phase Transition
}

\author{
Kelby K. Chan, Moshe Deutsch, ${ }^{(a)}$ B. M. Ocko, P. S. Pershan, and L. B. Sorensen ${ }^{(b)}$ \\ Division of Applied Sciences, Harvard University, Cambridge, Massachusetts 02138 \\ (Received 22 October 1984)
}

The temperature dependence of the square of the smectic order parameter, $|\psi|^{2}$, was determined from the integrated $x$-ray-scattering intensity. The data are described very well by the form $I(t) / I(0)=1 \mp A^{( \pm)}|t|^{x}$, where $t=\left(T-T_{N A}\right) / T_{N A}$. The determined values for $x$ do not agree with the theoretically expected value $x=1-\alpha$, where $\alpha$ is the heat-capacity exponent. This disagreement raises questions concerning the quantitative validity of the Landau-de Gennes free energy.

PACS numbers: 61.30.- $-\mathrm{v}, 61.10 .-\mathrm{i}, 64.60 . \mathrm{Fr}$

In spite of considerable research activity over the past ten years the nematic-to-smectic- $A(N-A)$ phase transition remains one of the principal unsolved problems in equilibrium statistical physics. ${ }^{1}$ Although the Landau free-energy density proposed by de Gennes in 1972 does explain most of the qualitative features of the transition, ${ }^{2}$ there are significant quantitative discrepancies between the current experimental results and the predictions based on this free energy. Recent experimental evidence of tricritical behavior in different materials may explain the apparent nonuniversality of critical exponents, ${ }^{3-5}$ however, there is still no consensus about either the universality class of the transition, or the effects of the inherent anisotropy of the critical correlations. Experiments that might improve our understanding have been hampered by the technical difficulty of maintaining well-oriented monodomain samples on the smectic side of the transition, and, except for heat-capacity ${ }^{6}$ and light scattering ${ }^{7}$ measurements, most of the experimental results are for the critical effects on the nematic side of the transition.

In this Letter we describe measurements of the integrated $x$-ray-scattering intensity which sum over the scattering from differently oriented domains. This is the classical crystallographers' solution ${ }^{8}$ to the problem of sample mosaic and it allows a quantitative measure of the mean square amplitude of the smectic order parameter, $\left\langle|\psi|^{2}\right\rangle$, on both the nematic and the smectic sides of the transition. One very general consequence of the Landau-de Gennes free energy is that $\left\langle|\psi|^{2}\right\rangle=L \mp M^{ \pm}|t|^{x}$, where $x$ is related to the heatcapacity exponent by $x=1-\alpha$ and $t=\left(T-T_{N A}\right) / T_{N A}$ ( $T_{N A}$ is the $N-A$ transition temperature). We show from the data that $x$ is not generally equal to $1-\alpha$ and therefore propose that the Landau-de Gennes free energy is not adequate for quantitative predictions regarding the $N-A$ transition.

The $\mathrm{x}$-ray-scattering spectrometer was configured to integrate over a large region of reciprocal space. ${ }^{9}$ A germanium monochromator crystal collimates the $\mathrm{Cu}$ $K \alpha \mathrm{x}$ rays in the scattering plane. A beam monitor between the monochromator and the sample is used to measure drifts in the incident beam intensity, and the data are normalized to a constant monitor level. The sample is held in a beryllium holder that is placed inside a two-stage oven capable of $\pm 0.5 \mathrm{mK}$ stability over $\sim 8 \mathrm{~h}$. Permanent cobalt-samarium magnets inside the oven provide a $4.3 \mathrm{kG}$ field that aligns the nematic director in the scattering plane parallel to the $z$ axis in the sample reference frame (see Fig. 1). A receiving slit $4 \mathrm{~mm}$ wide by $8 \mathrm{~mm}$ high, located $270 \mathrm{~mm}$ from the sample, is placed at $2 \theta=2 \sin ^{-1}(\lambda / 2 d)$, where $d$ is the smectic layer spacing and $\lambda(=1.542 \AA)$ is the $\mathrm{Cu} K \alpha$ wavelength. The vector $\mathrm{Q}_{0}=(2 \pi / d) \hat{\mathbf{z}}$. For $\theta=1.5^{\circ}$, the height of the spectrometer resolution function (dotted line in Fig. 1) corresponds to $\Delta Q_{z} / Q_{0}=0.15$. The crosshatched region indicates the $(x-z)$ cross-sectional area in the sample reciprocal space that is swept out by the resolution function when

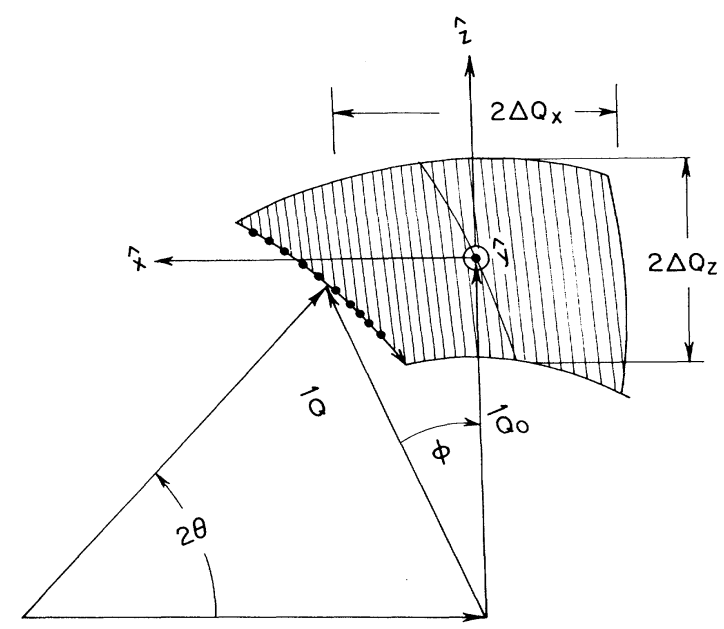

FIG. 1. Scattering geometry for the measurement of integrated intensity. The shaded region is the $(x-z)$ cross section of the reciprocal-space volume swept out by the resolution function as the sample reference frame is rotated from $-\phi$ to $\phi$. 


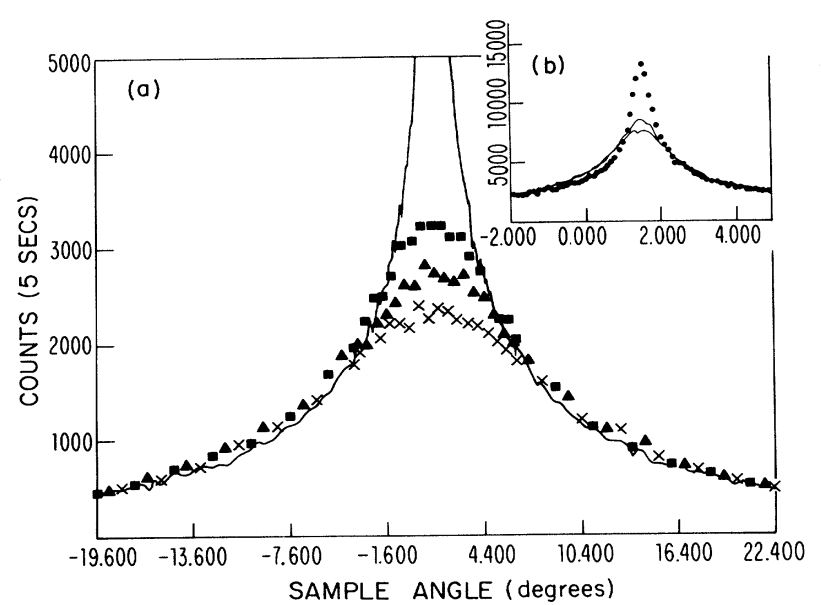

FIG. 2. Intensity vs sample rotation $\phi$ for $80 \mathrm{OB}$; $T_{N A}=66.205^{\circ} \mathrm{C}$. (a) Solid line, $T=66.214^{\circ} \mathrm{C}$; squares, $66.560^{\circ} \mathrm{C}$; triangles, $66.832^{\circ} \mathrm{C}$, and crosses, $67.234^{\circ} \mathrm{C}$. (b) Dots, $T=66.203^{\circ} \mathrm{C}$; solid lines, $66.208^{\circ} \mathrm{C}$ and $66.211^{\circ} \mathrm{C}$. (a) Displays only every sixth data point.

the sample is rotated by $\phi$. The thickness of the spectrometer resolution function (normal to the scattering plane) is $\Delta Q_{y} / Q_{0}=0.30$.

Figure 2(a) displays the measured scattering intensity versus sample orientation $\phi$ for octyloxycyanobiphenyl (8OCB) at different temperatures. The sample oven limits $|\phi|$ to $\leq 20^{\circ}$. The intensity obtained by integrating the $\phi$ scan has the effect of sweeping the spectrometer resolution over a region $\Delta Q_{x} / Q_{0}$ $=\sin \left(20^{\circ}\right)=0.34$. Therefore, the integrated intensity $I(t)$ is proportional to $\int_{\Delta^{3} Q} S(\mathbf{Q}) d^{3} Q$, where $S(\mathbf{Q})$ is the static structure factor and, for small $\theta$, the resolution volume $\Delta^{3} Q$ is approximately a rectangular parallelpiped $\left(2 \Delta Q_{x}\right)\left(2 \Delta Q_{y}\right)\left(2 \Delta Q_{z}\right)$.

Figure 3 displays $I(t) / I(0)$ for six materials. With the assumption that the error in each point is deter-

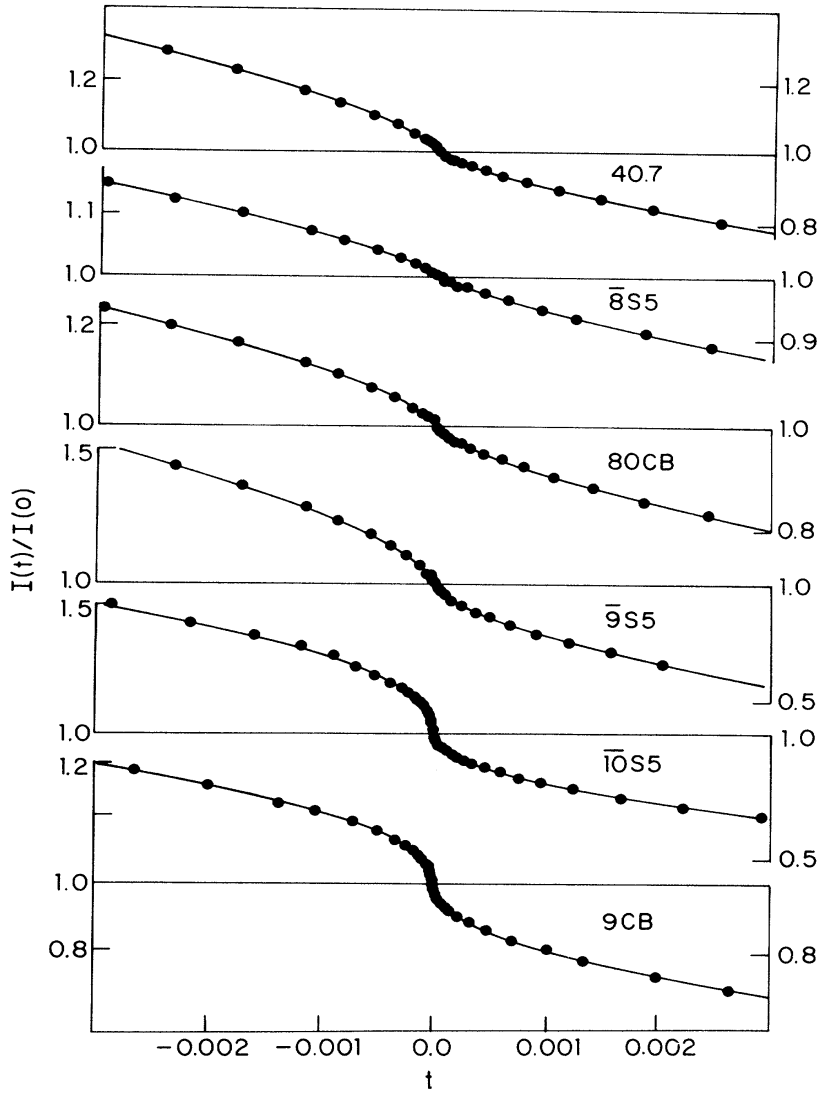

FIG. 3. Integrated intensity $I(t) / I(0)$. The solid lines are the results of nonlinear least-squares fits described in Table I.

mined by counting statistics, one standard deviation corresponds to $\sim 0.2 \%$ uncertainty in $I(t)$. Table $I$ displays the results of fitting the data by the form $I(t) / I(0)=1 \mp A^{ \pm}|t|^{x}$. 10 Except for the case of 10S5, independent fits of the data for $T<T_{N A}$ result

TABLE I. Results of nonlinear least-squares fits for the data in Fig. 3. Values of $\alpha$, $\nu_{11,1}$, and $\gamma$ are obtained from Refs. 4, 5, and 17. The last column is the empirically motivated scaling relation $x_{\mathrm{sc}}=\left(\nu_{\|}+\nu_{\perp}\right) / 2$. 40.7, 4- $n$-butyloxybenzylidene-4' $n$ heptylaniline; $\overline{8} \mathrm{~S} 5,4$ - $n$-pentylphenylthiol-4'-n-octyloxybenzoate; $\overline{9} \mathrm{~S} 5,4$ - $n$-pentylphenylthiol-4'-n-nonyloxybenzoate; $10 \mathrm{~S} 5,4-n$-pentylphenylthiol-4'- $n$-decyloxybenzoate; 9CB, 4cyano-4'-n-nonylbiphenyl.

\begin{tabular}{lcccccccc}
\hline \hline Material & $T_{N A} / T_{N I}$ & $A^{+} / A^{-}$ & $x$ & $\alpha$ & $\nu_{\perp}$ & $\nu_{\|}$ & $\gamma$ & $x_{\text {sc }}$ \\
\hline 40.7 & 0.926 & 0.69 & $0.70 \pm 0.05$ & -0.03 & 0.65 & 0.78 & 1.46 & 0.72 \\
8S5 & 0.936 & 0.90 & $0.76 \pm 0.05$ & 0.0 & 0.68 & 0.83 & 1.53 & 0.76 \\
8OCB & 0.963 & 0.87 & $0.68 \pm 0.05$ & 0.2 & 0.58 & 0.71 & 1.32 & 0.65 \\
9S5 & 0.967 & 0.73 & $0.63 \pm 0.05$ & 0.22 & 0.57 & 0.71 & 1.31 & 0.64 \\
10S5 & 0.984 &..- & $0.50 / 0.43^{\mathrm{a}}$ & 0.45 & 0.51 & 0.61 & 1.10 & 0.56 \\
9CB & 0.994 & 0.99 & $0.47 \pm 0.05$ & 0.53 & 0.37 & 0.57 & 1.10 & 0.47 \\
\hline
\end{tabular}

$$
{ }^{\mathrm{a}} x_{+} / x_{-} \cdot
$$


in $x^{+}=x^{-}$within experimental error. We discuss 10S5 below. The solid lines in Fig. 3 display the results of the fits for $\left|T-T_{N A}\right|<1 \mathrm{~K}$. The transition temperature, $T_{N A}$, can be identified with a precision of $\sim \pm 3 \mathrm{mK}$ without any numerical analysis. For example, Fig. 2(b) displays the relevant scans for 8OCB at $66.211,66.208$, and $66.203^{\circ} \mathrm{C}$. The first two scans are nematic and the wings superpose perfectly. The last scan is smectic and is easily recognized by the changes in the wings and a sudden decrease in the full width at half maximum. In addition, there is often a slight shift in the peak position at the transition. If $T_{N A}$ is allowed to vary over a reasonable range of values (i.e., $\pm 3 \mathrm{mK})$ the exponent, $x$, varies by less than approximately \pm 0.01 .

To understand the significance of these results, consider the definition of the structure factor

$$
S(\mathbf{Q})=(2 \pi)^{-3} \int\langle\delta \rho(\mathbf{r}) \delta \rho(0)\rangle \exp (-i \mathbf{Q} \cdot \mathbf{r}) d^{3} r,
$$

where $G(\mathbf{r})=\langle\rho(\mathbf{r}) \delta \rho(0)\rangle$ is the correlation function for density fluctuations. Let $H(\mathbf{Q})=1$ if $\mathbf{Q}$ is inside the shaded region in Fig. 1 and zero elsewhere. Then the integrated intensity

$$
\begin{aligned}
I(t) & =\int S(\mathbf{Q}) H(\mathbf{Q}) d^{3} Q \\
& =\int h(\mathbf{r})\langle\delta \rho(\mathbf{r}) \delta \rho(0)\rangle d^{3} r,
\end{aligned}
$$

where $h(\mathbf{r})=(2 \pi)^{-3} \int H(\mathbf{Q}) \exp (-i \mathbf{Q} \cdot \mathbf{r}) d^{3} Q$ is $10-$ calized to a region in real space of size $(2 \pi)^{3} / \Delta^{3} Q$ centered around the origin. If we substitute $\delta \rho(r)$ $=\operatorname{Re}\left[\psi \exp \left(+i Q_{0} z\right)\right]$, and assume ${ }^{11}$

$$
\begin{aligned}
G(\mathbf{r}) & =\operatorname{Re}\left[\left\langle\psi(r) \psi(0)^{*}\right\rangle\right] \\
& =C_{0}(\mathbf{r}) \mp|t|^{x} C_{ \pm}\left(\mathbf{r}_{\perp} / \xi_{\perp}, z / \xi_{\|}\right),
\end{aligned}
$$

it follows that since $\left(\xi_{\|} \Delta Q_{z}\right)^{-1}$ and $\left(\xi_{\perp} \Delta Q_{x, y}\right)^{-1}$ vanish as $t \rightarrow 0$,

$$
I(t)=L \mp M^{ \pm}|t|^{x},
$$

where

$$
L=\int C_{0}(\mathbf{r}) h(\mathbf{r}) d^{3} r
$$

and

$$
\begin{aligned}
M^{ \pm} & =\int C_{ \pm}\left(\mathbf{r}_{\perp} / \xi_{\perp}, z / \xi_{\|}\right) h(\mathbf{r}) d^{3} r \\
& =C_{ \pm}(0,0) \int h(\mathbf{r}) d^{3} r .
\end{aligned}
$$

For each data set the stability of the results were tested by range shrinking in which points furthest from the transition temperature were successively discarded and the data refitted. The absence of any clear trend due to range shrinking together with the fact that the bulk of the temperature dependence of $I(t)$ results from the central portion of the scan (i.e., $Q_{x}$ $<0.1 \Delta Q_{x}$ ) indicates that the resolution volume $\Delta^{3} Q$ is large enough to obtain all of the critical intensity.
The uncertainty in the critical exponent $x=0.68$ \pm 0.05 includes statistical errors, variations accompanying the range-shrinking process, and the uncertainty in $T_{N A}$.

If we take the asymptotic form for $G(\mathrm{r})_{t=0}$ $=C_{0}(\mathbf{r})=\left(r_{\perp}\right)^{-\left(1+\eta_{\perp}\right)}$ or $(z)^{-\left(1+\eta_{\|}\right)}$and note that for $t>0$ and for large $r$ the correlations must fall off faster than $(r)^{-(1+\eta)}$, then the leading terms in $C_{+}\left(\mathbf{r}_{\perp} / \xi_{\perp}, z / \xi_{\|}\right)$must cancel the algebraic decay. This requires forms for $C_{+} \sim\left(\xi_{\perp} / r_{\perp}\right)^{1+\eta_{\perp}}$ or $\left(\xi_{\|} / z\right)^{1+\eta_{\|}}$. To effect cancellation over a range of $t$, both $t^{x} \xi_{\|}^{\left(1+\eta_{\|}\right)}$and $t^{x} \xi_{\perp}^{\left(1+\eta_{\perp}\right)}$ must be temperature independent. Since $\xi \sim t^{-\nu}$, this requires that $x=\nu_{\|}\left(1+\eta_{\|}\right)=\nu_{\perp}\left(1+\eta_{\perp}\right)$. For the observed anisotropic exponents $\left(\nu_{\|} \neq \nu_{\perp}\right)$ this result is not consistent with the previous result of anisotropic scaling: $\gamma=\nu_{\|}\left(2-\eta_{\|}\right)=\nu_{\perp}\left(2-\eta_{\perp}\right){ }^{1} \quad$ Furthermore, since $\nu_{\perp}<x=\gamma / 2<\nu_{\|}$the pair of relations involving either $\nu_{\|}, \eta_{\|}$or $\nu_{\perp}, \eta_{\perp}$ are not individually satisfied. However, it is interesting to observe that within experimental error, the following empirical generalizations of isotropic scaling (with $\eta=0), x_{\mathrm{sc}}=\left(\nu_{\|}+\nu_{\perp}\right) / 2$, and $\gamma=\left(\nu_{\|}+\nu_{\perp}\right)$, are very well satisfied. ${ }^{12}$

There is a general argument ${ }^{13,14}$ that predicts $x=1-\alpha$ for the Landau-de Gennes free energy. The simplest form of the argument starts with the free energy $U=\int\left[a^{\prime} t|\psi|^{2}+\delta U(\psi)\right] d^{3} r$, and calculates the partition function $Z=\int \exp \left(-U / k_{\mathrm{B}} T\right) d \psi$. Differentiation with respect to temperature then yields the excess entropy $\Delta S \sim\left\langle|\psi|^{2}\right\rangle$. Since $\left\langle|\psi|^{2}\right\rangle \sim|t|^{x}$ the critical part of the heat capacity $C \sim t^{-\alpha} \sim t^{x-1}$, so that $x=1-\alpha$. The data in Table I show unambiguously that this reduction is not universally satisfied.

In view of this direct relation between the Landau free-energy density and the relation $x=1-\alpha$, the discrepancy suggests that the Landau-de Gennes form is missing some essential physics. One possibility is the existence of a nearby tricritical point. ${ }^{3-5}$ Unfortunately, the data in Table I show that the discrepancy is most serious for materials with the smallest McMillan ratio, ${ }^{15} T_{N A} / T_{N I}$, and these are the materials which should be furthest from the tricritical point. In fact the best agreement is for 9CB which is believed to be right at a tricritical point.

A second possibility concerns the data for $T<T_{N A}$. Even though the smectic- $A$ phase has only quasi-longrange order (QLRO), the integrated intensity measured in this low-resolution experiment might behave similarly to systems with true long-range order (TLRO). However, in either case (QLRO or TRLO) $\int S(\mathrm{Q}) d^{3} Q \sim\left\langle|\psi|^{2}\right\rangle \sim|t|^{1-\alpha}$. If the system had TLRO, $S(\mathrm{Q})$ would have one term proportional to $|\langle\psi\rangle|^{2} \delta\left(\mathbf{Q}-\mathbf{Q}_{0}\right)$ and a second diffuse term, with a different temperature dependence related to $\left\langle(\delta \psi)^{2}\right\rangle$. In the case of TLRO scaling theory defines $\beta$ such that $|\langle\psi\rangle|^{2} \sim|t|^{-2 \beta}$ with $2 \beta=2-\gamma-\alpha .^{16}$ Since for the 
data in Table I, $\gamma>1$, if the system were behaving like one with TRLO it would have $2 \beta<1-\alpha$. Thus if the measurement did not include all of the fluctuations, so that the measured value of $\left\langle(\delta \psi)^{2}\right\rangle$ was less than the true value, the data would yield an effective exponent $2 \beta<x^{\text {eff }}<1-\alpha$. We discount effects of this kind since independent fits of the data for $T \gtrless T_{N A}$ obtain equal values for $x^{ \pm}$and the extracted values of $x^{-}$do not vary with the range-shrinking process.

A third possibility is that $C_{ \pm}(0,0)=0$. Since $M$ must remain finite for all $t$, the only possibility is that $M \sim|t|^{\delta x}$, where $\delta x>0$. In this case the measured exponent $x^{m}=x+\delta x$ would be greater than $1-\alpha$. In fact, the measured values are les than or equal to $1-\alpha$. A final possibility is that because of chemical impurities, surface effects, or some other mechanism, the transitions are slightly first order. In this case, the data for $I(t)$ would be asymmetric about $t=0$ with a steeper slope on the smectic side. Again, since $x^{+}=x^{-}$, we discount this for every material, except 10S5. For $10 \mathrm{~S} 5$, however, other measurements ${ }^{4,5}$ indicate that it is near to a tricritical point and we believe that trace impurities have induced a first-order transition in the present $\overline{10} 5$ sample.

If we ignore the possibility that $x$ is only equal to $1-\alpha$ in a critical regime that is restricted to a range of $\left|T-T_{N A}\right|$ smaller than probed here, our present understanding of the critical properties predicted by the Landau-de Gennes free energy suggests that this free energy is not completely adequate. This is particularly distressing since the deficiencies are most serious for the materials, such as 40.7 and $8 \mathrm{~S} 5$, which were expected to be described best. These have the smallest McMillan ratios, heat-capacity exponents $\alpha=0$, and are furthest from a possible tricritical point.

We gratefully acknowledge discussions with B. Halperin and D. Nelson. This work was supported by the National Science Foundation under Grants No. DMR-82-12189 and No. DMR-80-20247.

(a) Permanent address: Physics Department, Bar-Ilan University, Ramat-Gan, Israel. (b) Permanent address: Department of Physics, FM-15, University of Washington, Seattle, Wash. 98105.

${ }^{1}$ For a recent review see T. C. Lubensky, J. Chim. Phys. 80, 31 (1983).

2P. G. de Gennes, Solid State Commun. 10, 753 (1972).

${ }^{3}$ D. Brisbin, R. De Hoff, T. E. Lockhart, and D. L. Johnson, Phys. Rev. Lett. 43, 1171 (1979).

${ }^{4}$ J. Thoen, H. Marynissen, and W. Van Dael, Phys. Rev. Lett. 52, 204 (1984).

5B. M. Ocko, R. J. Birgeneau, J. D. Litster, and M. E. Neubert, Phys. Rev. Lett. 52, 208 (1984).

${ }^{6}$ See Ref. 1 for a description of relevant experimental studies.

${ }^{7}$ M. R. Fisch, P. S. Pershan, and L. B. Sorensen, Phys. Rev. A 29, 2741 (1984), and Phys. Rev. Lett. 48, 943 (1982).

${ }^{8}$ See, for example, B. E. Warren, X-Ray Diffraction (Addision-Wesley, Reading, Mass., 1969), Chap. 4.

9J. Als-Nielsen, R. J. Birgeneau, M. Kaplan, J. D. Litster, and C. R. Safinya, Phys. Rev. Lett. 39, 352 (1977).

${ }^{10}$ Addition of a linear term, $B t$, to this form reduces the resultant values of $x$ by less than 0.02 with no significant improvement in the quality of the fit.

${ }^{11}$ The assumption that $G(\mathrm{r})=C_{0}(r) \mp|t|{ }^{x} C_{ \pm}(r / \xi)$ is a general consequence of isotropic scaling [see $M$. E. Fischer and A. Aharony, Phys. Rev. Lett. 31, 1238 (1973)]. The anisotropic form used here is a natural consequence of the anisotropic scaling hypothesis. The only feature of this form that is essential to the present discussion is the assumption that the product of the coefficient in the leading term in the temperature expansion of $G(\mathbf{r})-C_{0}(\mathbf{r})$ and the resolution function $h(\mathbf{r})$ are integrable.

12P. S. Pershan and J. Als-Nielsen, Phys. Rev. Lett. 52, 759 (1984).

${ }^{13}$ The most explicit statement of this result appears in Fischer and Aharony, Ref. 11, Eq. (4); however, general arguments supporting it are given by their several references.

${ }^{14} \mathrm{~A}$ similar form was introduced by Daves et al. for the hexatic order parameter near the hexatic-to-smectic- $A$ transition. S. C. Daves, J. Budai, J. W. Goodby, and R. Pindak, "X-Ray Study of the Hexatic- $B$ to Smectic- $A$ Phase trañsition in Liquid Crystal Films" (to be published).

15W. McMillan, Phys. Rev. A 7, 1419 (1973).

16H. Eugene Stanley, Introduction to Phase Transitions Critical Phenomena (Oxford Univ. Press, New York, 1971), p. 185.

${ }^{17}$ C. W. Garland, M. Meichle, B. M. Ocko, A. R. Kortan, C. R. Safinya, L. J. Yu, J. D. Litster, and R. J. Birgeneau, Phys. Rev. A 27, 3234 (1983). 